\title{
Edge-on disk around the T Tauri star [MR81] H $\alpha 17$ NE in Corona Australis *
}

\author{
R. Neuhäuser ${ }^{1}$, S. Krämer ${ }^{1}$, M. Mugrauer ${ }^{1}$, R. Köhler ${ }^{2,3}$, T. O. B. Schmidt ${ }^{1}$, \\ M. Ammler-von Eiff ${ }^{4}$, J. Alves $^{5}$, S. Fiedler ${ }^{1}$, and N. Vogt ${ }^{6,7}$
}

\author{
1 Astrophysikalisches Institut und Universitäts-Sternwarte, Friedrich-Schiller-Universität Jena, Schillergässchen 2-3, 07745 Jena, \\ Germany \\ e-mail: rne@astro.uni-jena.de \\ 2 ZAH Landessternwarte, Königstuhl, 69117 Heidelberg, Germany \\ 3 Max-Planck-Institut für Astronomie, Königstuhl 17, 69117 Heidelberg, Germany \\ Centro de Astronomia e Astrofísica da Universidade de Lisboa, Tapada da Ajuda, 1349-018 Lisboa, Portugal \\ 5 Calar Alto Observatory, Centro Astronomico Hispano-Aleman, C/q Jesus Durban Remon 2-2, 04004 Almeria, Spain \\ 6 Departamento de Física y Astronomía, Universidad de Valparaíso, Avenida Gran Bretaña 1111, Valparaíso, Chile \\ 7 Instituto de Astronomía, Universidad Catolica del Norte, Avda. Angamos 0610, Antofagasta, Chile \\ Received 1 August 2008 / Accepted 14 November 2008
}

\section{ABSTRACT}

\begin{abstract}
Aims. Using the speckle camera SHARP at the $3.5 \mathrm{~m}$ ESO NTT, Köhler and collaborators found an object $\sim 3.5$ mag fainter in $K$ only $1.3^{\prime \prime}$ north-east of the T Tauri star [MR81] $\mathrm{H} \alpha 17$ in the Corona Australis (CrA) star-forming region, which could be either a brown dwarf or a T Tauri star with an edge-on disk. We attempt to study this faint object in detail.

Methods. We acquired deep VLT NACO near-infrared images at three epochs to determine, whether [MR81] H $\alpha 17$ and the nearby faint object are comoving and to measure the infrared colors of both objects. We obtained optical and infrared spectra of both objects with the VLT using FORS and ISAAC, respectively, to determine spectral types and temperatures as well as ages and masses.

Results. The T Tauri star [MR81] H $\alpha 17$ and the faint nearby object have a projected separation of 1369.58 mas, i.e. $178 \mathrm{AU}$ at $130 \mathrm{pc}$. They share the same proper motion $(\sim 5 \sigma)$, so that they most certainly form a bound binary pair. The apparently fainter component [MR81] H $\alpha 17 \mathrm{NE}$ has a spectral type of M2e, while the apparently brighter component [MR81] H $\alpha 17 \mathrm{SW}$, the previously known T Tauri star, has a spectral type of M4-5e. We can identify a nearly edge-on disk around [MR81] H $\alpha 17$ NE by visual inspection, which has a diameter of at least 30 to $50 \mathrm{AU}$. We are able to detect strong emission lines in [MR81] H $\alpha 17 \mathrm{NE}$, which are almost certainly due to ongoing accretion. The NE object is detectable only by means of its scattered light.

Conclusions. If both objects are co-eval (2-3 Myr) and located at the same distance ( $130 \mathrm{pc}$ as $\mathrm{CrA})$, then the apparently fainter [MR81] $\mathrm{H} \alpha 17 \mathrm{NE}$ is more massive (primary) component with a nearly edge-on disk and the apparently brigther component [MR81] $\mathrm{H} \alpha 17 \mathrm{SW}$ is less massive (companion). Both are low-mass T Tauri stars with masses of $\sim 0.5$ and $0.23 \pm 0.05 M_{\odot}$, respectively.
\end{abstract}

Key words. astrometry - stars: binaries: visual - stars: formation - stars: pre-main sequence

\section{Introduction: [MR81] $\mathrm{H} \alpha 17$ in CrA}

The star-forming region Corona Australis ( $\mathrm{R} \mathrm{CrA}$ or CrA) harbors dozens of young intermediate- to low-mass stars (and brown dwarfs) from spectral types B8 down to M 8.5. They have an age of between one and a few Myr and at a distance of appoximately 130 pc (see Neuhäuser \& Forbrich 2008, for a recent review).

The first few low-mass, pre-main sequence stars, so-called $\mathrm{T}$ Tauri stars (TTS) in this star-forming region were found by early $\mathrm{H} \alpha$ and infrared (IR) imaging surveys by Knacke et al. (1973), Glass \& Penston (1975), and Marraco \& Rydgren (1981). The latter found an emission-line object, nowadays called [MR81] $\mathrm{H} \alpha 17$ (MR81 for Marraco \& Rydgren 1981, sometimes also [MR81] HA 17). It is located at $\alpha=$ $19^{\mathrm{h}} 10^{\mathrm{m}} 43.4^{\mathrm{s}}$ and $\delta=-36^{\circ} 59^{\prime} 09^{\prime \prime}$ for J2000.0. The catalogs GSC, USNO, NOMAD, DENIS, and 2MASS provide BVRIJHK magnitudes for the unresolved binary.

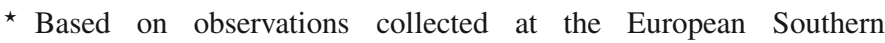
Observatory, Chile in runs 67.C-0213, 71.C-0206(D), 71.C-0522(B), 073.C-0164(A), 073.C-0167(B) and 081.C-0393(A).
Patten (1998) determined the spectral type M3-5 for the unresolved binary. The USNO B1.0 and NOMAD catalogs provide a proper motion of $2.0 \pm 25.0 \mathrm{mas} / \mathrm{yr}$ in right ascension and $-44.0 \pm 13.0 \mathrm{mas} / \mathrm{yr}$ in declination, while Ducourant et al. (2005) indicate a proper motion of $10 \pm 12 \mathrm{mas} / \mathrm{yr}$ in right ascension and $-24 \pm 12 \mathrm{mas} / \mathrm{yr}$ in declination. We decided to use the weighted mean, i.e. $8.5 \pm 10.8 \mathrm{mas} / \mathrm{yr}$ in right ascension and $-33.2 \pm 8.8 \mathrm{mas} / \mathrm{yr}$ in declination, which is consistent with kinematic membership to CrA (see e.g. Neuhäuser et al. 2000).

Using the speckle camera SHARP at the ESO $3.5 \mathrm{~m}$ NTT on 6 July 2001, Köhler et al. (2008) detected a faint object close to [MR81] $\mathrm{H} \alpha$ 17. The apparently fainter object is $\sim 3.5 \mathrm{mag}$ fainter in $\mathrm{K}$ and located at a separation of $\sim 1.3^{\prime \prime} \mathrm{NE}$ from the apparently brighter object [MR81] $\mathrm{H} \alpha 17$, which we now label [MR81] H $\alpha 17 \mathrm{SW}$; the apparently fainter object is now refered to as [MR81] $\mathrm{H} \alpha 17 \mathrm{NE}$. Figure 3 of Köhler et al. (2008) displays their SHARP $K$-band image of [MR81] H $\alpha 17 \mathrm{NE} \& \mathrm{SW}$, which has lower angular resolution and a lower signal-to-noise (S/N) ratio than our new VLT AO image (Fig. 1).

The apparently fainter object can be a young star with (nearly) edge-on disk, a brown dwarf companion, or an unrelated background star, as speculated by Köhler et al. (2008). We 


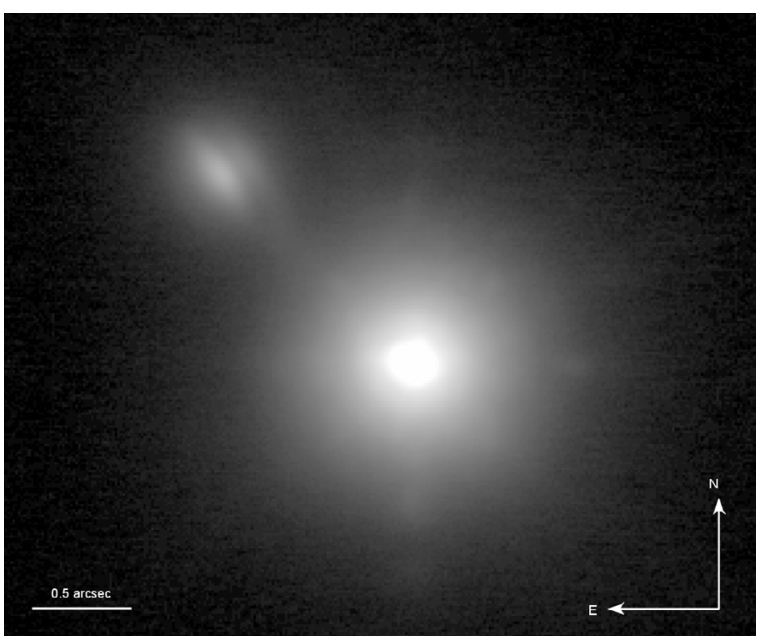

Fig. 1. VLT NACO $K$-band image of [MR81] H $\alpha 17 \mathrm{SW}$ (brighter object, $K=9.60 \mathrm{mag}$ ) and NE (top left, $K=13.24 \mathrm{mag}$ ) obtained in June 2004 with the nearly edge-on disk. This disk is seen as dark lane extended by about 200 to 450 mas (26 to $57 \mathrm{AU}$ at $130 \mathrm{pc}$ ) along the NE-SW direction. The dark lane thickness is $\sim 100$ mas, i.e. 15 AU (possibly flared disk). The SE surface of the disk reflects far more star light than the NW surface; hence, the disk is not exactly edge-on, but inclined by about $20^{\circ}$ from edge-on. Angular resolution and $\mathrm{S} / \mathrm{N}$ are insufficient for determining the disk properties accurately. The faint dark lane of the edge-on disk can be seen better on a computer screen when using the original fits file (http://www . astro.uni-jena.de/Users/ rne/cra-disk). See Fig. 2 for a 3D contour plot. See Fig. 13 in the Appendix for a color-composite $J H K$ image.

identify the fainter object as an early M-type T Tauri star with a nearly edge-on disk and strong emission lines due to ongoing accretion. We present the observations and data reduction in Sect. 2 and the results in Sect. 3.

\section{Observations and data reduction}

The apparently fainter object next to [MR81] H $\alpha 17$ was discovered on the $K$-band speckle images taken with SHARP-I (System for High Angular Resolution Pictures number I) of the Max-Planck-Institut for Extraterrestrial Physics (Hofmann et al. 1992) at the $3.5 \mathrm{~m}$ New Technology Telescope (NTT) of the European Southern Observatory (ESO) on La Silla, Chile (see Köhler et al. (2008) for details on the observations, data reduction, and the resulting image $)^{1}$. To find out whether the apparently fainter object could be either a sub-stellar companion or a star with an edge-on disk, follow-up observations were necessary.

Follow-up imaging in $J H K$ was completed one year later with the Infrared Array Camera and Spectrograph (ISAAC) of the ESO Very Large Telescope (VLT) on Cerro Paranal, Chile. Images of higher angular resolution and higher $\mathrm{S} / \mathrm{N}$ in $J H K$

\footnotetext{
1 The SHARP data were reduced independently by Köhler et al. (2008) with the speckle interferometry technique and by Krämer (2008) with a simple shift+add technique; they determined and used two slightly different pixel scales and detector orientations (Krämer uses $50.44 \pm$ $0.64 \mathrm{mas} / \mathrm{pixel}$ for right ascension, $48.02 \pm 0.64 \mathrm{mas} /$ pixel for declination, and a detector orientation of $90.6 \pm 0.5^{\circ}$ obtained by Ott (priv. comm.) from the Galactic Center images of the same night; see Table 1 for the astrometric solution of Köhler et al. (2008); with slightly different calibrations, the results on separation and PA also differ slightly; in this paper, we use the mean of their results for [MR81] $\mathrm{H} \alpha$ 17, listed in Table 2.
}

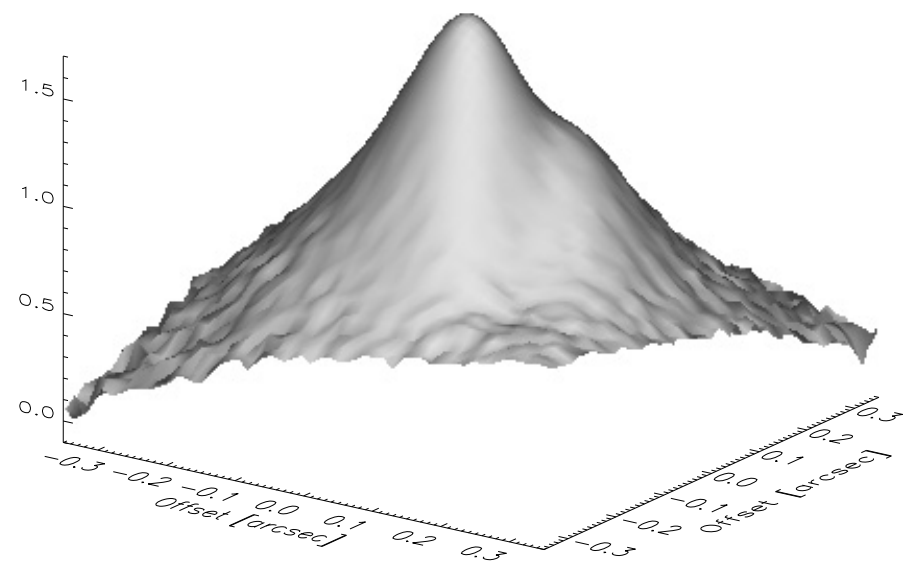

Fig. 2. Three-dimensional contour plot (log stretch) of the $K$-band brightness of [MR81] H $\alpha 17$ NE obtained from Fig. 1. The nearly edgeon disk, hardly visible as a dark lane in Fig. 1, is visible here as a step. A higher resolution image may be taken in the future with new AO instruments or larger telescopes.

were taken with the Adaptive Optics imager NACO (for NAOS CONICA for Nasmyth Adaptive Optics System, NAOS, with COude NearInfrared Camera and Array, CONICA; Rousset et al. 2003) at the ESO VLT in 2003, 2004, and 2008, with the S13 camera, i.e. a $14^{\prime \prime} \times 14^{\prime \prime}$ field of view. Optical and nearinfrared spectra of the apparently fainter object were obtained with the ESO VLT instruments FORS1 (FOcal Reductor and Spectrograph number 1) and ISAAC, respectively (see Table 1 for the entire observations $\log$ ).

All science and flat field frames acquired were dark and/or bias corrected, then the science frames were divided by a normalized flat field. A shift+add procedure was applied to subtract the background and to add up all frames in each filter for each observing run. The same procedure was performed for spectroscopic (telluric) and imaging (photometric and astrometric) standard stars. Spectra were wavelength calibrated with lamp arc exposures, divided by the telluric standard, and multiplied by a corresponding spectral template. For astrometric calibration of the imaging observations, we used astrometric standards observed in the same night, such as stars close to the Galactic Center or well-known Hipparcos binaries. Resulting pixel scales and detector orientations are given in Table 1; the errors include the effects of Gaussian centering errors in the science targets and the astrometric standards as well as possible motion in the standards (see e.g. Neuhäuser et al. (2008) for details of typical astrometric calibration procedure). For data reduction, we used ESO eclipse and MIDAS for imaging data, and IRAF for spectra.

\section{Results}

We present and discuss the results for our imaging (photometry and astrometry) data and spectroscopy (physical parameters) data.

\subsection{Imaging results: photometry and astrometry}

In our NACO images (Fig. 1), the ellipsoidal shape of the NE component is visible with a faint dark lane across the object. This image clearly resembles the typical appearence of a classical T Tauri star with (nearly) edge-on disk. 
Table 1. Observations log.

\begin{tabular}{|c|c|c|c|c|c|c|c|c|c|}
\hline $\begin{array}{l}\text { Telescope/ } \\
\text { instrument }\end{array}$ & $\begin{array}{r}\text { Observing } \\
\text { date }\end{array}$ & $\begin{array}{l}\text { Filter and } \\
\text { obs. mode }\end{array}$ & $\begin{array}{r}\text { DIT } \\
{[\mathrm{s}]}\end{array}$ & NDIT & NINT & $\begin{array}{l}F W H M \\
{[\mathrm{mas}]}\end{array}$ & $\begin{array}{c}\text { Pixel scale } \\
{[\mathrm{mas} / \mathrm{px}]}\end{array}$ & $\begin{array}{c}\text { Detector orientation } \\
{\left[^{\circ}\right] \text { (a) }}\end{array}$ & Remarks \\
\hline NTT/SHARP & 6-July-2001 & $K$ imaging & 0.5 & 250 & 2 & 671 & $49.5 \pm 0.2$ & $90.2 \pm 0.2$ & (b) \\
\hline VLT/ISAAC & 27-July-2002 & $J$ imaging & 1.8 & 33 & 7 & 652 & $147.856 \pm 0.047$ & $179.96 \pm 0.01$ & (c) \\
\hline VLT/ISAAC & 27-July-2002 & $H$ imaging & 1.8 & 33 & 7 & 801 & $147.856 \pm 0.047$ & $179.96 \pm 0.01$ & (c) \\
\hline VLT/ISAAC & 27-July-2002 & $K$ imaging & 1.8 & 33 & 7 & 717 & $147.856 \pm 0.047$ & $179.96 \pm 0.01$ & (c) \\
\hline VLT/FORS1 & 27-Mar.-2003 & red spectrum & 790 & (d) & 3 & - & 200 & 0 & (e) \\
\hline VLT/NACO & 4-Sept.-2003 & $K$ imaging & 1.7 & 40 & 10 & 83 & $13.24 \pm 0.02$ & $-0.02 \pm 0.09$ & (g) \\
\hline VLT/ISAAC & 5-May-2004 & $K$ spectrum & 0.11 & 9 & 26 & - & 71 & 0 & (e) \\
\hline VLT/NACO & 25-June-2004 & $K$ imaging & 3.5 & 20 & 27 & 116 & $13.23 \pm 0.05$ & $0.14 \pm 0.25$ & (f) \\
\hline VLT/NACO & 13-June-2008 & $J$ imaging & 20 & 3 & 22 & 391 & $13.243 \pm 0.056$ & $0.73 \pm 0.40$ & (g) \\
\hline VLT/NACO & 13-June-2008 & $H$ imaging & 15 & 4 & 30 & 285 & $13.243 \pm 0.056$ & $0.73 \pm 0.40$ & (g) \\
\hline VLT/NACO & 13-June-2008 & $K$ imaging & 10 & 6 & 30 & 231 & $13.243 \pm 0.056$ & $0.73 \pm 0.40$ & (g) \\
\hline
\end{tabular}

Remarks: total exposure time are DIT (individual Detector Integration Times) times NDIT (number of DITs, together saved in one fits file) times NINT (number of jitter positions and files). (a) Measured from North over East to South. Positive detector orientations mean that they are to be added to values measured on raw frames. (b) Speckle imaging reported in Köhler et al. (2008), $500 \times 0.5 \mathrm{~s}$ on two detector positions each. For astrometric calibration, see footnote 1. (c) Astrometric calibration with 2MASS sources in the field-of-view. (d) Three spectra of $790 \mathrm{~s}$ exposure each. (e) Nominal pixel scale and detector orientation from the fits header. (f) Astrometric calibration from Neuhäuser et al. (2005). (g) Astrometry done as in Neuhäuser et al. (2008) with Galactic Center images for 2003 and images of the Hipparcos binary HIP 73357 for 2008.

Table 2. Astrometric results.

\begin{tabular}{lccc}
\hline \hline Date & $\begin{array}{c}\text { Separation } \\
{[\mathrm{mas}]}\end{array}$ & $\begin{array}{c}\text { PA } \\
{\left[{ }^{\circ}\right]}\end{array}$ & Remark \\
\hline 6-July-2001 & $1313 \pm 32$ & $45.15 \pm 2.0$ & (a) \\
27-July-2002 & $1384 \pm 39$ & $45 \pm 1.9$ & (b) \\
4-Sept.-2003 & $1369.5 \pm 7.3$ & $45.07 \pm 0.32$ & (b) \\
25-June-2004 & $1365.8 \pm 3.9$ & $45.4 \pm 0.25$ & (b) \\
13-June-2008 & $1373.4 \pm 7.2$ & $45.88 \pm 0.40$ & (b) \\
\hline
\end{tabular}

Remarks: (a) Means from data in Krämer (2008) and Köhler et al. (2008) using slightly different SHARP pixel scales. (b) this work.

The first young stellar object displaying this pattern, HH 30 IRS in Taurus, was imaged by the Hubble Space Telescope (Burrows et al. 1996). Edge-on disks are also observable with ground-based near-IR imaging observations both with AO, e.g. HK Tau/c (Stapelfeldt et al. 1998; Koresko 1998), HV Tau C (Monin \& Bouvier 2000), and LkH $\alpha 263$ C (Jayawardhana et al. 2002) and seeing-limited, e.g. 2MASSI $\mathrm{J} 1628137243139$ (Grosso et al. 2003). Hence, this object is most probably a young star with a nearly edge-on disk. The faint red object also resembles the appearance of so-called infrared companions, see e.g. Koresko \& Leinert (2001), a typical example of which is T Tau Sa (Koresko et al. 2000).

Astrometric and photometric data for NE are obtained after subtraction of the Point Spread Function (PSF) of the brighter star SW. Our astrometric results for the objects [MR81] $\mathrm{H} \alpha 17$ SW \& NE are listed in Table 2. The brighter object (SW) has a $F W H M$ of $116 \pm 4$ mas. The position angle (PA) of the disk major axis is $47.0 \pm 0.2^{\circ}$, where the extension along the disk lane axis is maximal, namely 247 mas, while the extension perpendicular to the disk lane is only 181 mas (both measured in the June 2004 NACO image); this disk angle is closely aligned (within $2^{\circ}$ ) with the PA between the SW and NE sources. However, as seen in Figs. 4 and 5, a more pole-on orbit of SW around NE is more likely than a more edge-on orbital inclination, so that disk and binary orbit were not coplanar.

The NACO image after subtraction of the PSF of SW is shown in Fig. 3. We detect an additional faint object $\sim 2$ " $\mathrm{NW}$ of [MR81] $\mathrm{H} \alpha 17 \mathrm{SW}$. This object is detected only in our highest quality image, the NACO image of June 2004, not in any other image. This faint object close to a bright star, for which only one

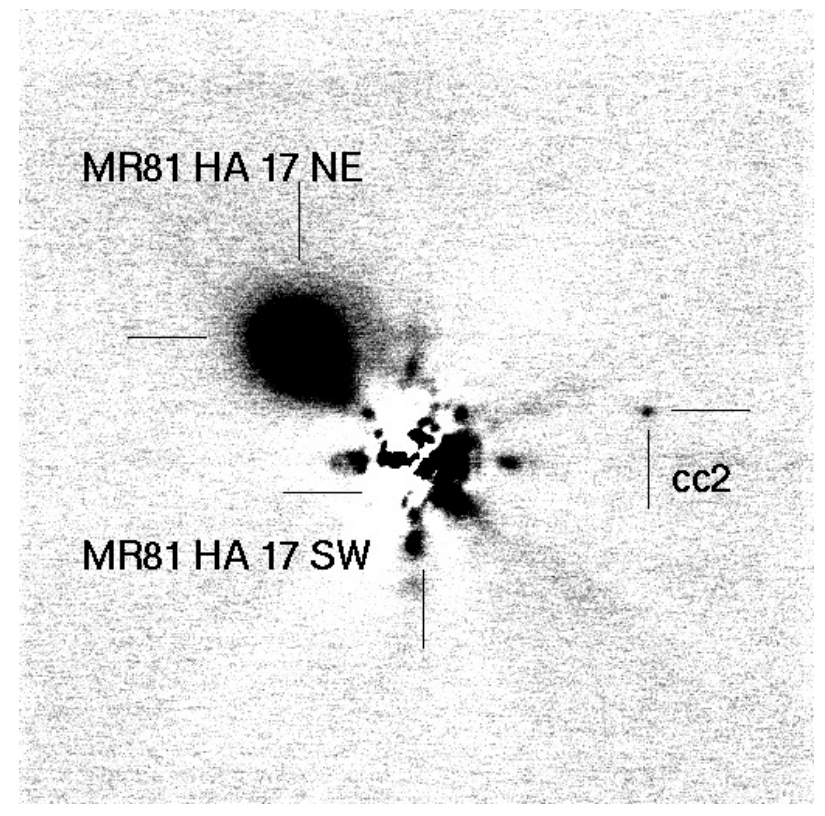

Fig. 3. VLT NACO $K$-band image of [MR81] $\mathrm{H} \alpha 17$ (obtained in June 2004) after subtraction of the PSF of the brighter object (SW), located here in the center of the image, the remaining speckles are seen as noise. One can see the fainter object NE (top left, $K=13.24$ mag, edge-on disk not seen here due to cuts selected to see $\mathrm{cc} 2$ ) and also another much fainter object towards to WNW (to the right, $K=18.56 \mathrm{mag}$ ), called cc2 here. This is a companion candidate, which would have only 1-2 Jupiter masses, if bound. However, we have only one epoch available for this faint object, because it is not detected in the other images.

epoch of data is available, must be regarded as a companion candidate (cc); we call it [MR81] $\mathrm{H} \alpha 17 \mathrm{SW} / \mathrm{cc} 2$ (cc1 was what is now [MR81] H $\alpha 17 \mathrm{NE}$ ). This new object is $1988.714 \pm 6.97$ mas west of SW and $420.1098 \pm 7.80$ north of SW, corresponding to $\sim 264$ AU projected separation at $130 \mathrm{pc}$. This separation is close to the separation between the stars [MR81] $\mathrm{H} \alpha 17 \mathrm{SW}$ and NE; such an non-hierarchical triple would probably be unstable. With $K=18.56 \pm 0.1 \mathrm{mag}$ (and using B.C. $K=3.3 \mathrm{mag}$ as for substellar L- and T-type objects), it would have a bolometric luminosity (at $130 \mathrm{pc}$ ) of $\log L_{\mathrm{bol}} / L_{\odot}=-4.7 \pm 0.1$ and, hence, only about 1 to 2 Jupiter masses (according to either Burrows et al. 1997; 


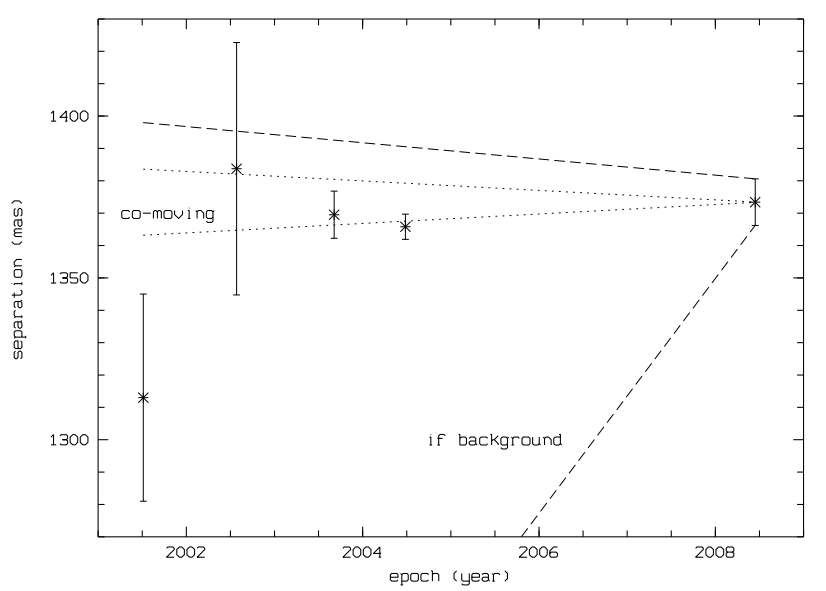

Fig. 4. Separation (in mas) versus observing epoch (in years) for data listed in Table 2. The dotted lines (starting from the 2008 data point opening to the past) indicate maximum possible separation change due to orbital motion in case of edge-on orbit. The dashed lines are for the background hypothesis, if the brighter object (SW) would have moved according to its (poorly) known proper motion, while the fainter object (NE) would be a non-moving object; we use the weighted mean of the USNO and NOMAD values for the proper motion of the SW object, namely $\mathrm{mu}_{\alpha}=8.5 \pm 10.8 \mathrm{mas} / \mathrm{yr}$ and $\mathrm{mu}_{\delta}=-33.2 \pm 8.8 \mathrm{mas} / \mathrm{yr}$; the two dashed lines also start in 2008 and open to the past, they take into account the error in proper motion. The last three data points from NACO with smallest error bars are consistent with (nearly) constant separation, the average being 1369.58 mas.

or Baraffe et al. 1998), for 2-3 Myr of age. However, not only are these models with evolutionary tracks uncertain at such very young ages, we also should note that this object is more likely to be a background object then another bound companion. A 2nd epoch image should be taken to check for common proper motion. In addition to NE and cc2, there are no other objects detected within $5^{\prime \prime}$ around SW down to $K=19 \mathrm{mag}$; faint objects within $0.5^{\prime \prime}$ of SW cannot be excluded.

In Figs. 4 and 5, we show the evolution in the separation and PA with time to investigate whether the NE and SW components form a common proper motion pair. For the epoch 2002 (ISAAC), we use only the $K$-band data, because the fainter object is hardly detected in $J$ and $H$.

To test the background and companion hypotheses, we estimate the maximum possible orbital motion for edge-on orbit (separation change) and pole-on orbit (PA change). We assume a circular orbit. The most reliably measured separation (2004) is $1365.8 \pm 3.9$ mas, i.e. $\sim 178 \mathrm{AU}$ at $130 \mathrm{pc}$, yielding a $\sim 3000 \mathrm{yr}$ long orbit (for $0.6 M_{\odot}$ total mass). When using all data available, common proper motion appears possible; only the SHARP data point regarding separation appears to be deviant by 1 to $2 \sigma$. When using only the NACO data, which provide the most accurate measurements, the smallest errors, and are all taken with the same instrument and same set-up (high angular resolution AO), both objects clearly exhibit common proper motion, i.e. are most certainly bound and orbiting each other.

Photometric results are as follows. By comparing aperture photometry for the apparently brighter object SW and the apparently fainter object NE (after subtraction of the PSF of SW), we derive magnitude differences as follows: $\Delta J \simeq 5.01 \mathrm{mag}$ (i.e. a factor of 101), $\Delta H \simeq 4.27 \mathrm{mag}$, and $\Delta K_{\mathrm{s}} \simeq 3.64 \mathrm{mag}$. Using in addition the error-weighted averages of the 2MASS and DENIS magnitudes for the unresolved object [MR81] $\mathrm{H} \alpha$ 17, we obtain $K_{\mathrm{s}}=13.24 \pm 0.05 \mathrm{mag}, H=14.4 \pm 0.05 \mathrm{mag}$, and $J=$ $15.96 \pm 0.05 \mathrm{mag}$ for the object NE , and $K_{\mathrm{s}}=9.60 \pm 0.02 \mathrm{mag}$,

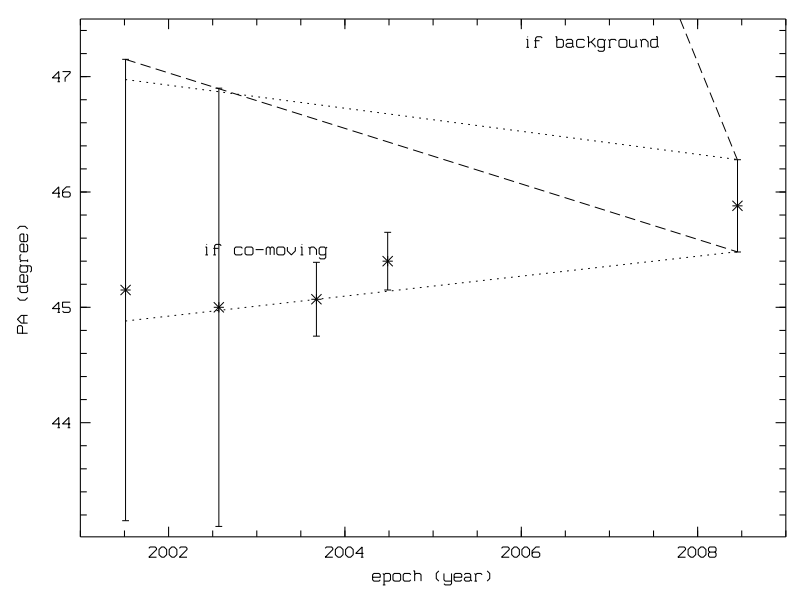

Fig. 5. Position angle (in degrees) versus observing epoch (in years) for data listed in Table 2. The dotted lines (starting from the 2008 datapoint and opening to the past) indicate maximum PA change due to orbital motion in the case of a pole-on orbit. The dashed lines indicate the background hypothesis, if the brighter object ( $\mathrm{SW}$ ) has moved according to its (poorly) known proper motion, while the fainter object (NE) would be a non-moving object; again, we use the weighted mean of the USNO and NOMAD proper motions for the SW object. The datapoints from 2003 and 2004 are inconsistent with the background hypothesis by $\sim 5 \sigma$ (together), so that in this case we can reject the background hypothesis. The datapoints are fully consistent with common proper motion.

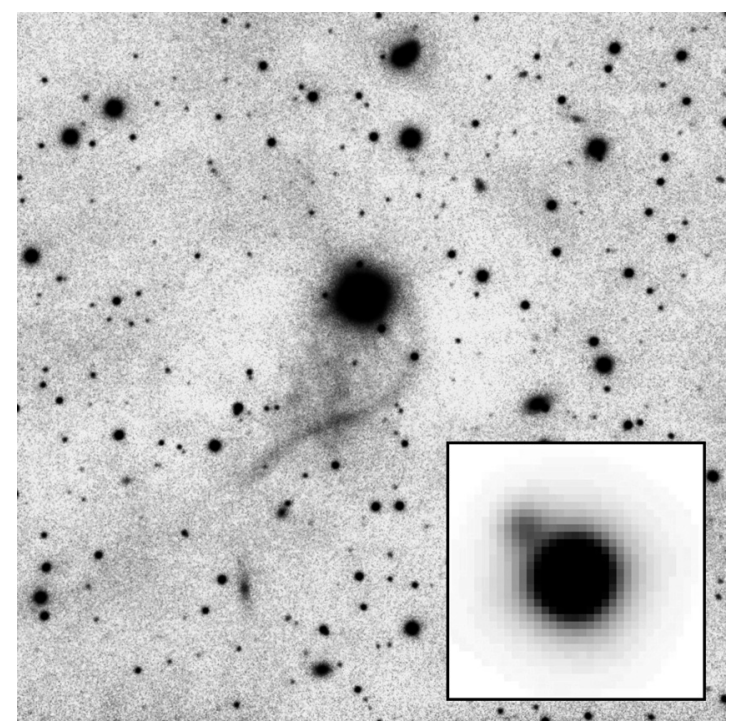

Fig. 6. Our VLT/ISAAC image of [MR81] $\mathrm{H} \alpha 17$ (central bright star) with the filamentary structure towards the south. The binary is unresolved in the main image due to cuts selected to see the filament, but seen when displayed with other cuts (lower right box with $5^{\prime \prime} \times 5^{\prime \prime}$ field of view). The main image field size is $126^{\prime \prime} \times 126^{\prime \prime}$, north is up, east is to the left. See Fig. 14 in the Appendix for a $J H K$ color composite; the image shown above is a contrast-enhanced $\mathrm{B} \& \mathrm{~W}$ version of the $J H K$ color composite.

$H=10.13 \pm 0.03 \mathrm{mag}$, and $J=10.96 \pm 0.01 \mathrm{mag}$ for the object SW.

In the ISAAC $J H K$ images, we observe a filament in addition to [MR81] $\mathrm{H} \alpha 17 \mathrm{SW}$ and NE (see Fig. 6), that is most pronounced in the $J$ and $H$-bands, but also visible in $K_{\mathrm{s}}$; it is also marginally visible in the 2MASS JHK images (lower sensitivity). Given the visual appearence, one is tempted to speculate that the filament originates in [MR81] $\mathrm{H} \alpha 17$; we observe a 
helix- or spiral-like structure going first towards SW for $\sim 14^{\prime \prime}$, then appears to curve to the SE (detected in our ISAAC images until 37" separation from [MR81] $\mathrm{H} \alpha$ 17, or 5000 AU at $130 \mathrm{pc}$ ); it is detected even out to $\sim 170^{\prime \prime}$ separation towards SE $(\sim 22360 \mathrm{AU}$ at $130 \mathrm{pc})$ in the 2MASS images. Strong (forbidden) emission lines, as detected in [MR81] $\mathrm{H} \alpha 17 \mathrm{NE}$, are often related to outflows, so that a physical connection is possible, but uncertain. The curvature could be produced by rotational and/or orbital revolution. However, the proper motion of the binary is directed towards the south, i.e. not away from the filament in the plane of the sky, but it is of course possible that the (as yet unknown) radial (or orbital?) velocity of [MR81] H $\alpha 17$ NE ensures that the filament trails behind. It would be important to complete a narrow-band imaging surbey of this feature and to monitor its possible variability.

\subsection{Spectroscopy results}

The optical spectrum was aqquired on 27 Mar. 2003 with VLT/FORS1 (three exposures of $790 \mathrm{~s}$ each at airmass 1.2 to 1.3) with the $0.7^{\prime \prime}$ slit and the order-sorting filter OG590 and grism 300I, i.e. covering $6000 \AA$ to $1.1 \mu \mathrm{m}$ with a dispersion of $2.5 \AA$ resulting in a spectral resolution of between 6.4 and $11 \AA$. The faint object NE was located directly on the slit, and the brighter object SW was also close to the slit, so that we could also extract its spectrum. The optical spectra are shown in Figs. 7 and 8.

In the optical spectrum, we observe remarkable emission lines with $\mathrm{H} \alpha$ being clearly the strongest of all. The remainder of the spectrum is dominated by strong emission of forbidden lines such as [OI], [NII], and [SII]. These forbidden lines are usually signs of shocked low-density regions of young stars such as outflows, winds, and jets (see e.g. Cabrit et al. 1990; Hamann 1994; Hartigan et al. 1995; Hirth et al. 1997; Dougados et al. 2000; and Fernández \& Comerón 2001). Outflow is often seen as both an indication and result of ongoing accretion.

The optical spectrum with these strong emission lines looks generally similar to young, low-mass objects like HBC 617 in Lupus (Krautter et al. 1984), LS-RCrA 1 in CrA (Fernández \& Comerón 2001; Comerón \& Fernández 2001), as well as Sz 102, Sz 106, and Par-Lup3-4 in Lupus (Comerón et al. 2003), e.g. our object [MR81] $\mathrm{H} \alpha 17 \mathrm{NE}$ has an $\mathrm{H} \alpha$ equivalent width of $\sim 330 \AA$ (but may be slightly saturated), while LS-RCrA 1 has 360 Å. Fernández \& Comerón (2001) concluded that LS-RCrA 1 is surrounded and extincted by an edge-on disk, which was not resolve by their imaging data.

We list all emission lines for which we care able to measure reliable equivalent widths in Table 3. Between 7319 and $7329 \AA$, the noise is too strong, and/or possible lines to weak, and/or spectral resolution too poor to unambiguously identify feature(s) either as [CaII] line at $7324 \AA$ or as a [OII] doublet at 7319 and $7329 \AA$. The CaII triplet at 8498 to $8662 \AA$ is detected.

The five strongest emission lines in [MR81] $\mathrm{H} \alpha 17 \mathrm{NE}$ are saturated, and their equivalent width measurements therefore represent uncertain limits. There even appear Fake emission lines even appear at these wavelengths in the other object [MR81] $\mathrm{H} \alpha 17 \mathrm{SW}$, which was located outside the slit. Therefore, it is also difficult to measure the $\mathrm{H} \alpha$ equivalent width of the object SW (whose upper limit should be $\sim 16 \AA$ ), and we are unable to classify unambiguously the SW object as being either a classical or weak-line T Tauri star. For the BVRIJHK part of the spectral energy distribution, the SW object resembles a blackbody; the IRAS 12 to $60 \mu \mathrm{m}$ data are offset above the

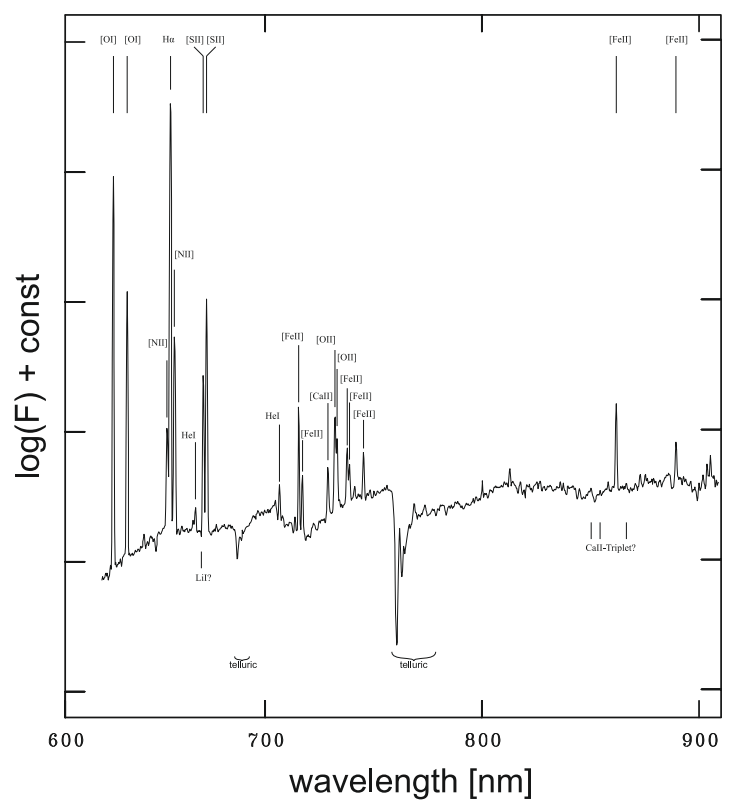

Fig. 7. Optical VLT/FORS1 spectrum of [MR81] H $\alpha 17$ NE with normalized flux versus wavelength (in $\mathrm{nm}$ ). Several strong emission lines are indicative of ongoing accretion.

Table 3. Lines detected in [MR81] H $\alpha 17$ NE.

\begin{tabular}{lccc}
\hline \hline Line & $\begin{array}{c}\lambda_{0} \\
{[\AA] \text { lab. }}\end{array}$ & $\begin{array}{c}\lambda \\
{[\AA] \text { obs. }}\end{array}$ & $\begin{array}{c}\text { equ. width } \\
{[\AA]}\end{array}$ \\
\hline$[\mathrm{OI}]$ & 6300 & 6301.0 & $-205:$ \\
{$[\mathrm{OI}]$} & 6364 & 6365.0 & $-69:$ \\
{$[\mathrm{NII}]$} & 6548 & 6548.6 & -11 \\
$\mathrm{H} \alpha$ & 6563 & 6564.1 & $-330:$ \\
{$[\mathrm{NII}]$} & 6583 & 6584.3 & -36 \\
$\mathrm{HeI}$ & 6678 & 6679.7 & -2 \\
{$[\mathrm{SII}]$} & 6716 & 6716.7 & $-21:$ \\
{$[\mathrm{SII}]$} & 6730 & 6731.1 & $-47:$ \\
$\mathrm{HeI}$ & 7066 & 7067.2 & -2 \\
{$[\mathrm{FeII}]$} & 7155 & 7156.3 & -13 \\
{$[\mathrm{FeII}]$} & 7452 & 7453.5 & -3 \\
{$[\mathrm{FeII}]$} & 8617 & 8618.3 & -9 \\
{$[\mathrm{FeII}]$} & 8892 & 8893.8 & -2 \\
\hline
\end{tabular}

Note: colons indicate uncertain limits due to saturation.

blackbody (see Fig. 10), but it is unclear, whether the far-IR emission originates in the immediate surroundings of the star (its disk) or its more distant surroundings (the star-forming cloud). The object [MR81] H $\alpha 17$ NE can clearly be classified as a classical T Tauri star because of its strong $\mathrm{H} \alpha$ emission and edge-on disk.

Due to poor spectral resolution, strong (saturated) nearby [SII] emission lines, and strong veiling in the NE object (and low S/N data of the SW object), the Lithium $6708 \AA$ line is detected only marginally in both the SW and NE components.

We determine the spectral types of both objects by comparison with $\mathrm{M}$ dwarfs of known spectral types from Kirkpatrick et al. (1991, see Fig. 8). Surprisingly, the spectral type of the apparently fainter component (NE) is earlier (M2e) than that of the apparently brighter component (SW), which is M4-5e according to our spectra. These spectral types are consistent with the results of Patten (1998), who assigned M3-5e to the unresolved system.

We display the $K$-band spectra of both objects in Fig. 9, both of which exhibit the CO lines typical of late-type objects. The 


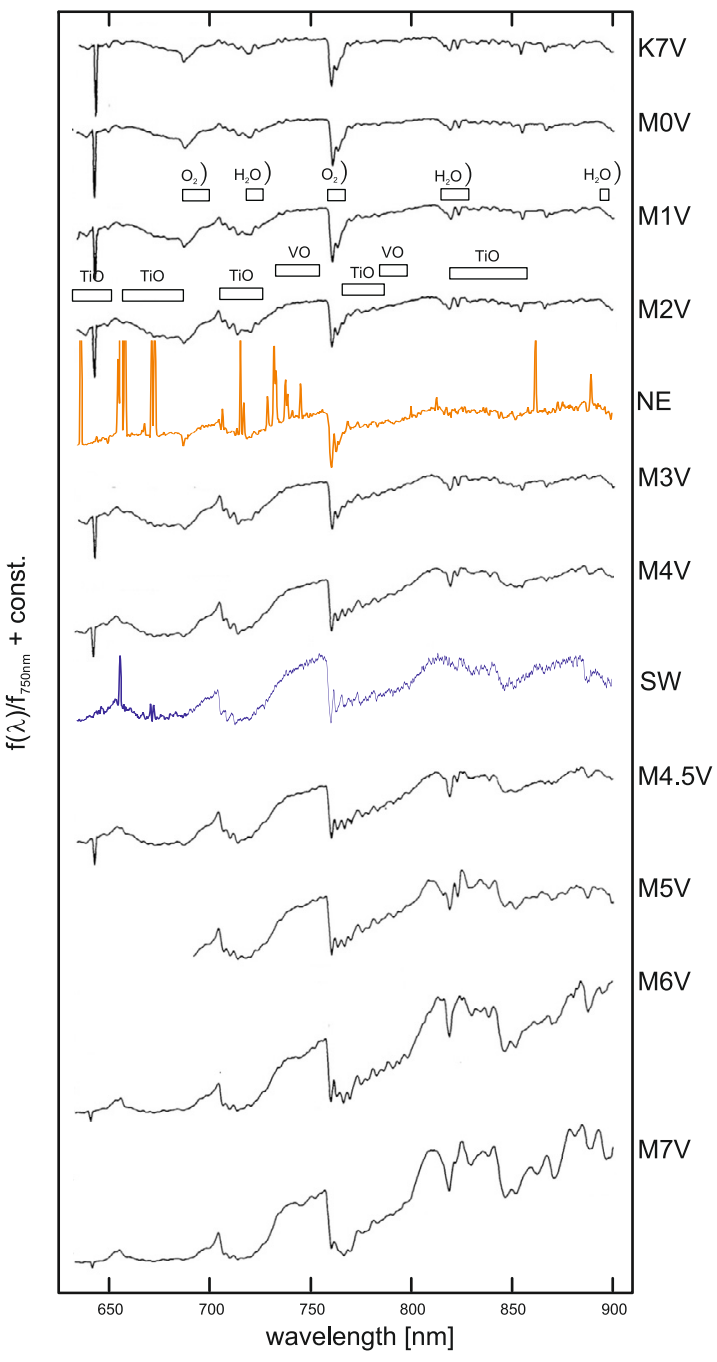

Fig. 8. Spectra of standard stars and [MR81] $\mathrm{H} \alpha 17 \mathrm{NE}$ and SW (marked NE and SW), normalized flux (shifted in y-axes to separate spectra) versus wavelength (in $\mathrm{nm}$ ). We obtain spectral types to be M2e for the apparently fainter object NE and M4-5e for the apparently brighter object SW. We indicate the location of telluric water and oxygen lines in the top row (between M0 and M2) and the location of detected molecular TiO and VO bands in the 2nd row (between M1 and M2).

spectra of [MR81] $\mathrm{H} \alpha 17 \mathrm{NE}$ also exhibit emission lines of $\mathrm{H}_{2}$ and $\operatorname{Br} \gamma$. As in LS-RCrA 1 (Fernández \& Comerón 2001), the $\mathrm{H}_{2}$ lines are stronger than the $\mathrm{Br} \gamma$ line, but in the data of our object, the $\mathrm{Br} \gamma$ line is clearly detected and there are more $\mathrm{H}_{2}$ emission lines detected than in LS-RCrA 1 . These $\mathrm{H}_{2}$ emission lines are also observed in other young low-mass objects surrounded by dense circumstellar material such as GY 11 (Greene \& Lada 1996; Wilking et al. 1999). Our VLT ISAAC $H$-band spectra of both objects are basically feature-less and triangular-shaped, as is typical of young M-type objects. According to both the $H$ and $K$-band spectra, the NE component is slightly earlier (early$\mathrm{M}$ ) in spectral type than the SW component (mid-M), as also observed in the optical spectra.

Since we detect indications of youth in both objects, such as emission lines and Lithium absorption, both objects are young and most probably CrA members; the proper motions of both objects are also consistent with CrA membership. If both objects are at the same distance and age, then the earlier-type component should be more massive and intrinsically brighter than the later-type object. Obviously, a large amount of flux from the NE

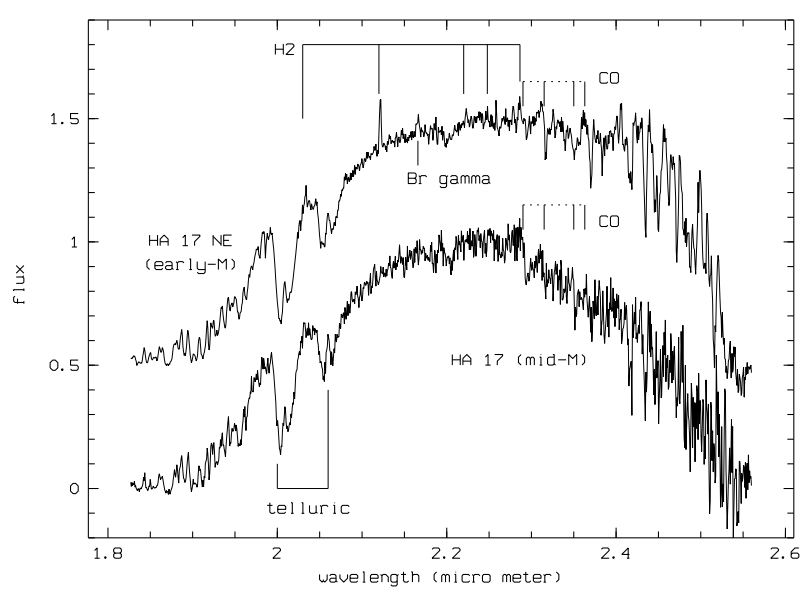

Fig. 9. Infrared VLT/ISAAC $K$-band spectra of [MR81] $\mathrm{H} \alpha 17 \mathrm{NE}$ (top) and $\mathrm{SW}$ (bottom), flux versus wavelength (in $\mu \mathrm{m}$ ), with $\mathrm{H} 2$ and $\mathrm{Br} \gamma$ emission lines in the object NE that indicate the accretion.

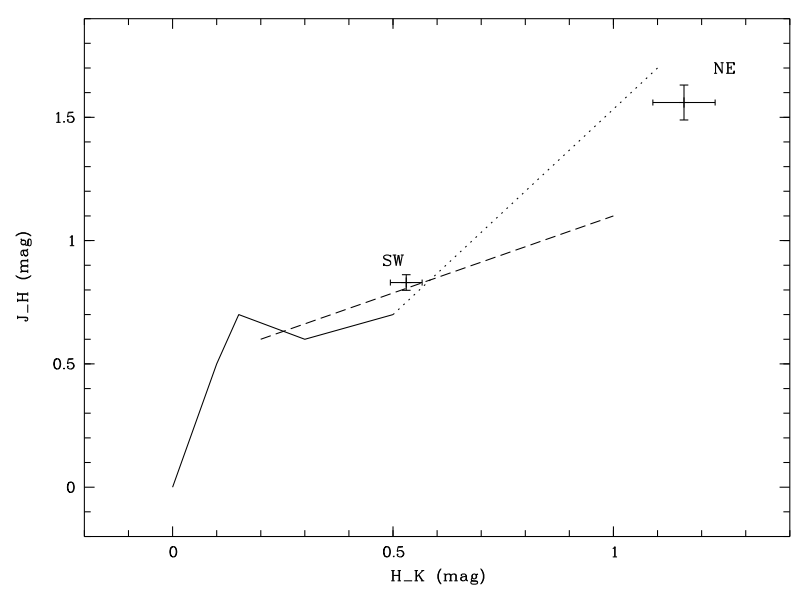

Fig. 10. JHK color-color diagram for [MR81] $\mathrm{H} \alpha 17 \mathrm{SW}$ and NE (plotting $J-H$ versus $H-K$, both in mag). The full curved line indicates the loci of unreddened M dwarfs down to M6 (Bessell \& Brett 1988); the dotted line is the reddening vector for 10 mag of extinction; and the dashed line indicates the loci of classical T Tauri stars (Meyer et al. 1997). Objects located to the right of the reddening vector are surrounded by circumstellar material producing near-IR excess emission. Our object SW is slightly extincted, but does not exhibit a near-IR excess. The fainter object NE, however, appears strongly extincted and also shows some near-IR excess, which is typical of T Tauri stars with disks.

component is absorbed by its edge-on disk, and the forbidden emission lines and the strong $\mathrm{H} \alpha$ emission are a clear characteristic of accretion.

\section{Discussion}

Given the spectral type and $J H K$ colors $^{2}$, we can estimate the extinction towards the $\mathrm{SW}$ object and obtain $A_{\mathrm{V}} \simeq 1$ mag for $\mathrm{SW}$; the extinction towards the NE objects cannot be determined, because we unablt to detect photospheric light.

In the $J H K$ color-color diagram, both objects lie on or close to the reddening vector, which has its origin at a mid-M dwarf

${ }^{2}$ Also optical colors $B V R I$ for the unresolved object from USNO, NOMAD, GSC, and DENIS, which are almost identical to the colors of the apparently brighter object [MR81] H $\alpha 17$ SW. 


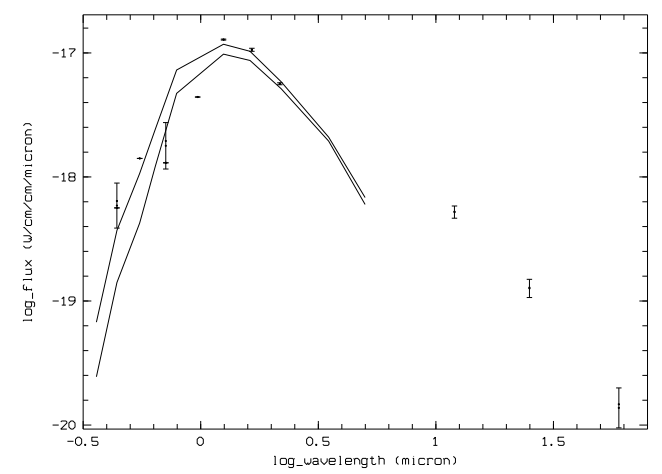

Fig. 11. Spectral energy distribution of [MR81] $\mathrm{H} \alpha 17 \mathrm{SW}$ with data from GSC, USNO, NOMAD, and this paper (error bars include measurement errors, when available, and variability) as well as IRAS together with M4 and M5 standard stars. We plot log of flux in $\mathrm{W} / \mathrm{cm}^{2} / \mu \mathrm{m}$ versus $\log$ of wavelength in $\mu \mathrm{m}$. The brighter object SW shows no near-IR excess. There is strong far-IR excess at the IRAS data points plotted here, but the spatial resolution of IRAS is not sufficient to conclude that $\mathrm{H} \alpha 17 \mathrm{SW}$ has IRAS excess emission; the IRAS data plotted here are for IRAS source F19073-3704 (Moshir et al. 1989).

(see Fig. 10). The object SW is located at the intersection of the reddening vector for dwarfs and the loci of classical T Tauri stars (Meyer et al. 1997), i.e. there is no evidence of near-IR excess emission from the SW component. The NE object lies 10 mag of extinction away from the unabsorbed mid-M dwarf and also about one tenth of a magnitude to the right (red) of the reddening vector, which indicates strong near-IR excess emission typical of circumstellar material (and a disk). See also Fig. 11 for the spectral energy distribution of [MR81] H $\alpha 17$ SW, which again shows its spectral type to be M4-5, but no near-IR excessemission.

We then calculate their bolometric luminosities (at $130 \mathrm{pc}$ ), obtaining $\log \left(L_{\mathrm{bol}} / L_{\odot}\right) \simeq-0.9 \pm 0.1$ for the apparently brighter SW $\left(\mathrm{M} 4-5 \mathrm{e}\right.$, hence $\left.T_{\text {eff }} \simeq 3220 \mathrm{~K}\right)$ and $\log \left(L_{\text {bol }} / L_{\odot}\right) \simeq-1.8 \pm$ 0.2 for the apparently fainter NE (M2e, hence $\left.T_{\text {eff }} \simeq 3560 \mathrm{~K}\right)$, using a temperature scale for young M-type objects that is intermediate between dwarfs and giants from Luhman (1999). However, since the faint NE object is detectable only in scattered light due to the edge-on disk, we are unable to study the stellar photosphere, so that the aforementioned luminosity for the object $\mathrm{NE}$ is not the luminosity of the star. This luminosity value appears strongly under-luminous, which it is typical of objects with edge-on disks, (see e.g. Watson \& Stapelfeldt 2007).

The SW object (M4-5e) lies on the 2-3 Myr isochrone of the Baraffe et al. (1998) models (see Fig. 12) and on the $3 \mathrm{Myr}$ isochrone on both the Burrows et al. (1997) and D'Antona \& Mazzitelli (1997) models. According to those models, it lies between the (interpolated) 0.15 to $0.30 M_{\odot}$ tracks. Hence, we obtain an age of $2-3 \mathrm{Myr}$ and a mass of $0.23 \pm 0.05 M_{\odot}$ for this object (SW). Given its temperature, the NE object lies on the $\sim 0.3$ to $0.5 M_{\odot}$ tracks, according to Baraffe et al. (1998) and D’Antona \& Mazzitelli (1997), almost independently of luminosity, which cannot be measured. Hence, the apparently fainter object (early-M) is more massive than the apparently brighter object (mid-M), $\sim 0.5 M_{\odot}$, if coeval with the SW object.

\section{Summary and conclusion}

Based on imaging and spectroscopic observations in the red optical and near-infrared, we conclude that the object [MR81] H $\alpha 17$ $\mathrm{NE}$ is surrounded (and absorbed) by a disk seen nearly edge-on.

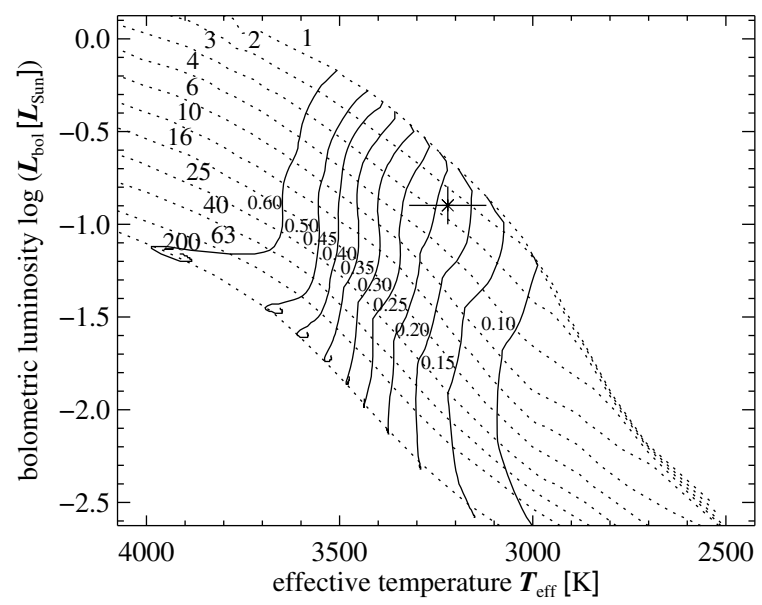

Fig. 12. H-R diagram with the location of [MR81] H $\alpha 17$ SW, bolometric luminosity versus effective temperature, compared to theoretical tracks (full lines with masses given in solar masses) and isochrones (as dashed lines with age given in Myr) from Baraffe et al. (1998), version with helium content $Y=0.275$, metallicity $[\mathrm{M} / \mathrm{H}]=0$, and mixing length $\alpha=1.0$. We conclude that SW has an age of 2-3 Myr and a mass of $\sim 0.23 M_{\odot}$. NE cannot be plotted here, because we see it only in reflected light, so that we cannot measure its luminosity.

The NE object appears fainter and has a spectral type M2e, while the object SW appears brighter, but has spectral type M4-5e. Both objects exhibit indicatons of youth such as emission lines and common proper motion with known CrA members, i.e. they are both low-mass T Tauri stars (both exhibit strong $\mathrm{H} \alpha$ emission).

The astrometric data from SHARP 2001 to NACO 2008 indicate that the two objects separated by $\sim 1.3^{\prime \prime}$ exhibit common proper motion. When using only AO data from NACO, i.e. data of the highest quality pixel scale and resolution, and aquired by an identical instrument, then we have strong evidence for common proper motion. Even with similar proper motion, they could be independent members of the young $\mathrm{CrA}$ star-forming region. In both cases, they have an age of 2-3 Myr at $\sim 130 \mathrm{pc}$.

A more detailed investigation of the emission lines is beyond the scope of this paper, and should be completed together with a study of their expected variability and higher-resolution spectra. Our spectral resolution is insufficient to resolve the line shape, e.g. of an inverse P Cyg profile, or to measure accurately outflow velocities. Using data of even higher resolution and/or deeper studies in the future, which cover a wider wavelength range, e.g. from space, one can investigate in detail the density distribution, scale height, and the gas and dust population, possibly polarization, accretion, outflow, variability, and ongoing planet formation in the disk.

Acknowledgements. We would like to thank the ESO User Support Group and ESO service mode observers for their support and observations. We have made use of the 2MASS, DENIS, USNO, GSC, NOMAD, and IRAS Faint Source Catalogs as well as the Simbad, Visir, and ADS databases. R.N. wishes to acknowledge general support from the German National Science Foundation (Deutsche Forschungsgemeinschaft, DFG), grants NE 515/13-1, 13-2 and 23-1. MAvE was supported first by a graduate scholarship of the Cusanuswerk, one of the national student elite programs of Germany, and then by an individual fellowship granted by the Fundação para a Ciência e a Tecnologia (FCT), Portugal (reference SFRH/BPD/26817/2006). TOBS acknowledges support from Evangelisches Studienwerk e.V. Villigst, another national student elite program in Germany. NV acknowledges support by grants FONDECYT 1061199 and DIPUV 07/2007. We also thank our referee, Karl Stapelfeldt, for very helpful remarks. 


\section{Appendix A}

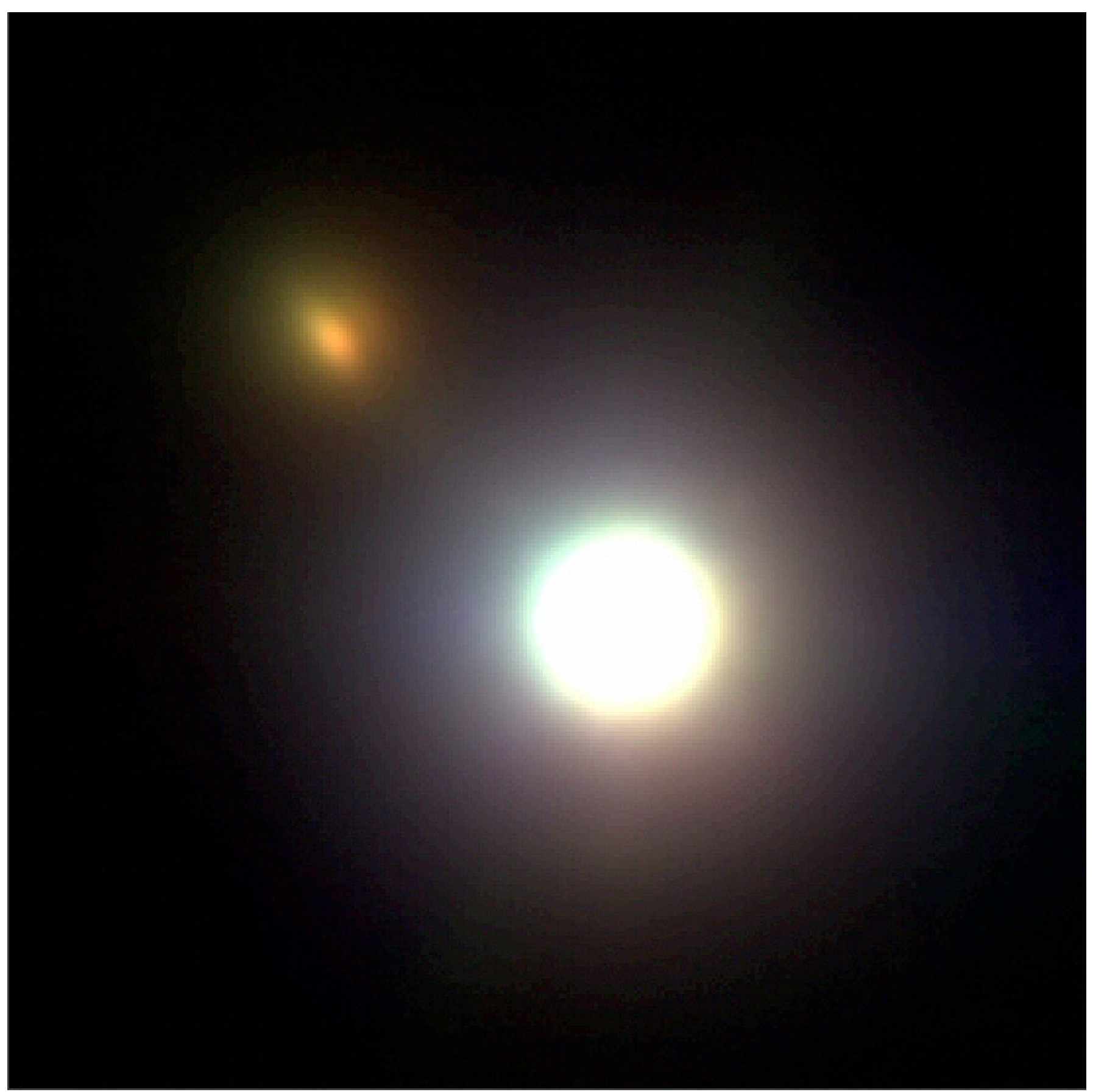

Fig. 13. JHK color composite of our VLT/NACO images of [MR81] H $\alpha 17 \mathrm{SW}$ (brighter object, $K=9.60 \mathrm{mag}$ ) and NE (top left, $K=13.24$ mag, separated $\sim 1.3^{\prime \prime}$ ) using the $J$ - and $H$-band images obtained in June 2008 and the $K$-band image obtained in June 2004 under better seeing conditions (than in June 2008). This nearly edge-on disk is seen as dark lane in front of the fainter object along the NE-SW direction. See Fig. 3 for a 3D contour plot. 


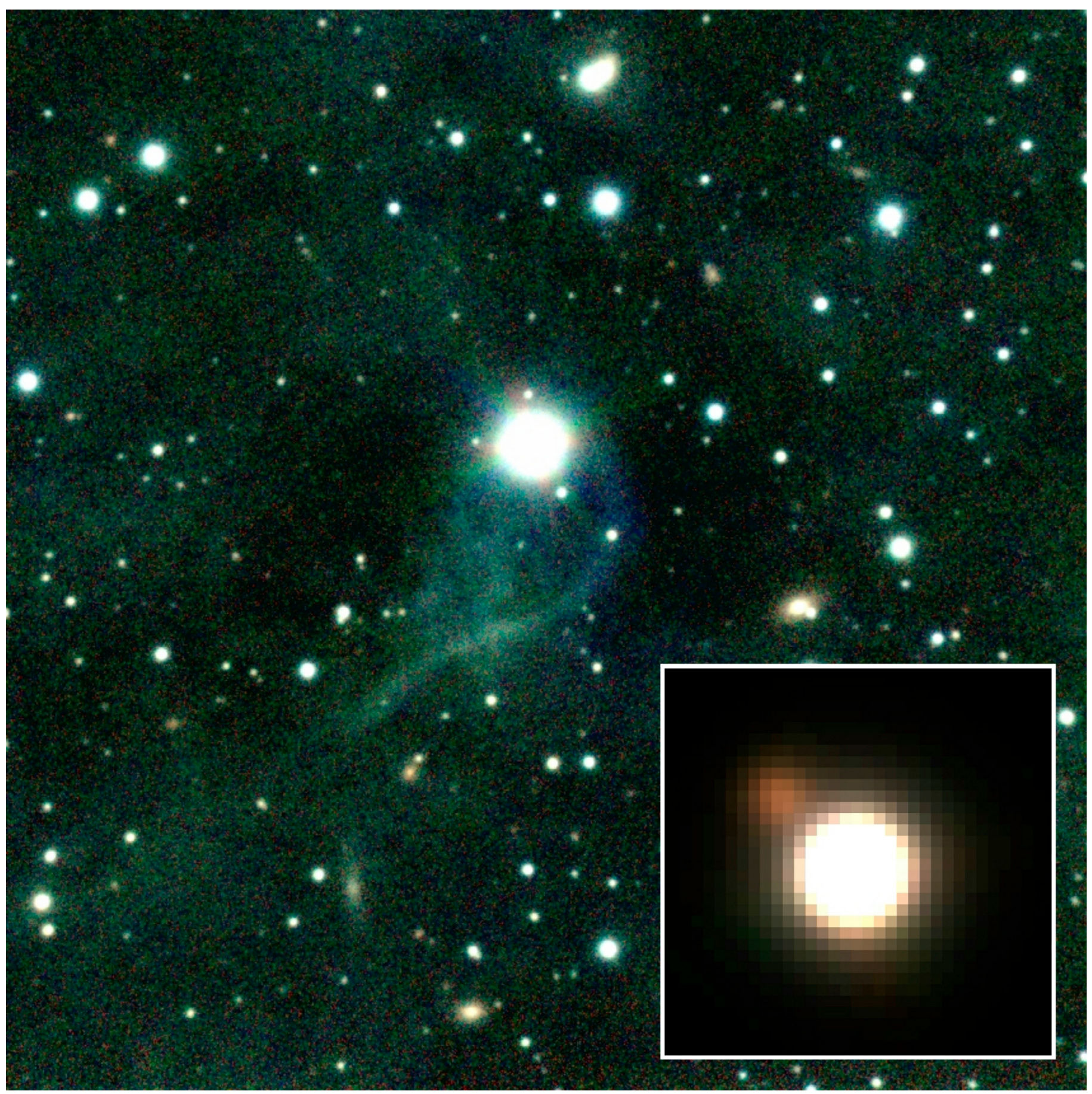

Fig. 14. JHK color composite of our VLT/ISAAC images of [MR81] H $\alpha 17$ (central bright star) with the filamentary structure towards the south. The binary is unresolved in the main image due to cuts selected to see the filament, but seen when displayed with other cuts (lower right box with $5^{\prime \prime} \times 5^{\prime \prime}$ field of view). The main image field size is $126^{\prime \prime} \times 126^{\prime \prime}$, north is up, east to the left.

\section{References}

Baraffe, I., Chabrier, G., Allard, F., \& Hauschildt, P. H. 1998, A\&A, 337, 403 Bessell, M. S., \& Brett, J. M. 1988, PASP, 100, 1134

Burrows, C. J., Stapelfeldt, K. R., \& Watson, A. M. 1996, ApJ, 473, 437

Burrows, A., Marley, M., Hubbard, W., et al. 1997, ApJ, 491, 856

Cabrit, S., Ferreira, J., \& Raga, A. C. 1990, A\&A, 343, L61

Comerón, F. \& Fernández, M. 2001, ESO Messenger, 103, 21

Comerón, F., Fernández, M., Baraffe, I., Neuhäuser, R., \& Kaas, A. A. 2003, A\&A, 406, 1001

D’Antona, F., \& Mazzitelli, I. 1997, MmSAI, 68, 807

Dougados, C., Cabrit, S., Lavally, C., \& Menard, F. 2000, A\&A, 357, L61

Ducourant, C., Teixeira, R., Perie, J. P., et al. 2005, A\&A, 438, 769

Fernández, M., \& Comerón, F. 2001, A\&A, 380, 264
Glass, I. S., \& Penston, M. V. 1975, MNRAS, 172, 227

Greene, T. P., \& Lada, C. J. 1996, AJ, 112, 2184

Grosso, N., Alves, J., Wood, K., et al. 2003, ApJ, 586, 296

Harmann, F. 1994, ApJS, 93, 485

Hartigan, P., Edwards, S., \& Ghandour, L. 1995, ApJ, 452, 736

Hirth, G. A., Mundt, R., \& Solf, J. 1997, A\&AS, 126, 437

Hofmann, R., Blietz, M., Duhoux, Ph., et al. 1992, in Progress in Telescope and Instrumentation Technologies, ESO Conference and Workshop Proceedings, ed. M. H. Ulrich (ESO Garching), 42, 617

Kirkpatrick, J. D., Herny, T. J., \& McCarthy, D. W. 1991, ApJS, 77, 417

Jayawardhana, R., Luhman, K. L., D’Alessio, P., \& Stauffer, J. R. 2002, ApJ, 571, L51

Köhler, R., Neuhäuser, R., Krämer, S., et al. 2008, A\&A, 488, 997 
Koresko, C. D. 1998, ApJ, 507, L145

Koresko, C. D. 2000, ApJ, 531, L147

Koresko, C. D., \& Leinert, C. 2001, in The Formation of Binary Stars, ed. H. Zinnecker, \& D. Mathieu, Proc. IAU Symp., 200, 265

Knacke, R. F., Strom, K. M., Strom, S. R., Young, E., \& Kunkel, W. 1973, ApJ 179,847

Krämer, S. 2008, Diploma Thesis, University Jena

Krautter, J., Reipurth, B., \& Eichendorf, W. 1984, A\&A, 133, 169

Luhman, K. L. 1999, ApJ, 525, 466

Marraco, H. G., \& Rydgren, A. E. 1981, AJ, 86, 62 (MR81)

Meyer, M. R., Calvet, N., \& Hillenbrandt, L. A. 1997, AJ, 114, 288

Monin, F. J., \& Bouvier, J. 2000, A\&A, 356, L75

Moshir, M. 1989, IRAS Faint Source Survey Catalog and explanatory supplement, IPAC
Neuhäuser, R., \& Forbrich, J. 2008, The Corona-Australis star forming region, in Handbook of Low Mass Star Forming Regions, Astron. Soc. Pacific (Invited Review), ed. B. Reipurth, 735

Neuhäuser, R., Walter, F. M., Covino, E., et al. 2000, A\&A, 146, 323

Neuhäuser, R., Guenther, E. W., Wuchterl, G., et al. 2005, A\&A, 435, L13

Neuhäuser, R., Mugrauer, M., Seifahrt, A., Schmidt, T., \& Vogt, N. 2008, A\&A, 484, 281

Patten, B. M. 1998, in The Tenth Cambridge Workshop on Cool Stars, Stellar Systems and the Sun, ed. R. A. Donahue, \& J. A. Bookbinder, ASP Conf. Ser., 154, 1755

Rousset et al. 2003, SPIE, 4839, 14

Stapelfeldt, K., Krist, J. E., Menard, F., et al. 1998, ApJ, 502, L65

Watson, A. M., \& Stapelfeldt, K. R. 2007, AJ, 133, 845

Wilking, B. A., Greene, T. P., \& Meyer, M. R. 1999, AJ, 117, 469 